\title{
Search for new physics with same-sign isolated dilepton events in CMS
}

\author{
Aniello Spiezia* \\ INFN and Universitá degli Studi di Perugia \\ on behalf of the CMS Collaboration \\ E-mail: aniello.spiezia@pg.infn.it
}

One of the main goals of LHC is the discovery of new physics (NP) beyond the standard model (BSM). Many strategies to get a hint of NP have been proposed and studied: one of these is the search for events in which two same-sign leptons are present. This topology is very rare in the Standard Model, while it is present in many BSM models. An inclusive search for the presence of new physics in events with two isolated same sign leptons, using $5.0 \mathrm{fb}^{-1}$ of data taken in 2011 by CMS is presented. The observed data are compatible with the predictions of the SM. The results are interpreted in terms of limits of a supersymmetric model.

Proceedings of the Corfu Summer Institute 2012 "School and Workshops on Elementary Particle Physics and Gravity"

September 8-27, 2012

Corfu, Greece

\footnotetext{
* Speaker.
} 


\section{Introduction}

An inclusive search for the presence of NP is presented in events with two isolated same sign leptons (SSDL), using the first $5.0 \mathrm{fb}^{-1}$ of certified CMS data taken in 2011 [1]. For a detailed description of the CMS detector at LHC see [2]. The analysis was performed for the search of same sign $e \tau, \mu \tau$ and $\tau \tau$ leptons, with high $p_{T}$ and in events with transverse missing energy and no requests on jet multiplicity. The production of two leptons with the same charge is very rare in the Standard Model, while it is present in many BSM models, as susy models [3] [4], models with heavy Majorana neutrinos [5] or models with fourth generation $d$-type quarks [6].

In CMS, several searches have been focused on the study of susy models with new heavy strongly interacting particles, that can be produced with large cross sections and hence could be easily seen with early LHC data [7]. On the contrary electroweak production of sparticles does not yield to large hadronic activity. In this case a cleaner final topology is present, but paying this feature by having lower cross sections. Here a simplified model, the chargino-neutralino pair production [8] is studied. In this model a chargino and a neutralino can be produced from a proton-proton collision and then decay giving a final state with three charged leptons, a neutrino and two neutralinos; the latter, being stable particles, elude the detector giving the missing energy of the event (together with the neutrino), see figure 1 . If the slepton $\tilde{\ell}$ and the neutralino $\tilde{\chi}_{2}^{0}$ have similar masses, one of the leptons can be produced with a low transverse momentum and can be not seen by the detector, so that in the final state there are two same sign leptons.

This search is focalized on the so-called $\tau$-enriched scenario, i.e. a scenario in which the chargino decays exclusively to $\tau$ leptons and the neutralino decays democratically, i.e. with the same probability to the three leptons. Considering this the SSDL final state will be: $p p \rightarrow e^{ \pm} \tau^{ \pm}, \mu^{ \pm} \tau^{ \pm}, \tau^{ \pm} \tau^{ \pm}+$ $M E T$, where $M E T$ stands for missing transverse energy and where the $\tau$ decay hadronically.

This final state topology search will be combined with the results from the three leptons with transverse mass topology results.

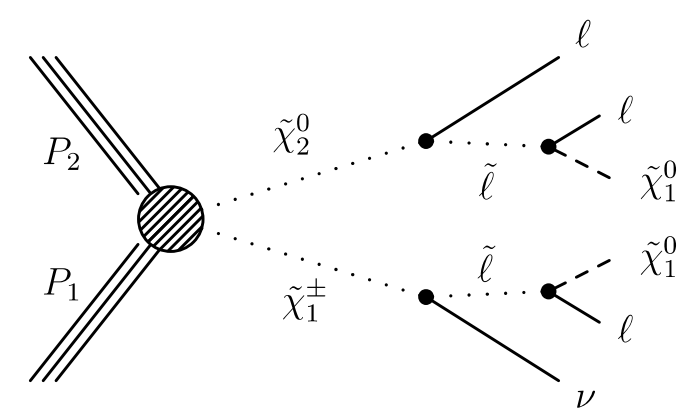

Figure 1: Chargino-neutralino pair production coming from a proton-proton collision and decaying in three charged leptons, a neutrino and two neutralinos.

\section{Experimental strategy}

Leptons that pass the selection can be divided in two categories: fake leptons are leptons that we considered as background and can both be objects that are wrongly reconstructed as leptons or genuine leptons from heavy-flavor decay; prompt leptons, that is leptons from $\mathrm{W}, \mathrm{Z}$ or NP particles 
decays, which are properly the signal we are looking for. From this definition, the following three sources of background can be defined and studied separately:

- Events with one real lepton and hadronic activity that mimics the $\tau$ (ttbar, $\mathrm{W}+\mathrm{jets}, \mathrm{QCD}$ ): this background is evaluated using the tight-to-loose method

- Events where opposite sign leptons are present ( $t \bar{t}$ or Drell-Yan events), but where the $\tau$ is reconstructed with the wrong charge (the electron and $\mu$ charge mis-identification rates are negligible compared to the $\tau$ one)

- Events with two prompt same sign leptons coming from SM processes; the main contribution is from the production of two gauge bosons that decay leptonically $(q \bar{q} \rightarrow W Z, Z Z)$

The signal region is defined requesting for high transverse missing energy, i.e. MET $>200 \mathrm{GeV}$. while the validation of the background evaluation methods is done in different regions of MET. In figure 2 this validation is shown, comparing the background prediction (for the three different background sources) with the observations. A good agreement is present for all the MET spectrum.

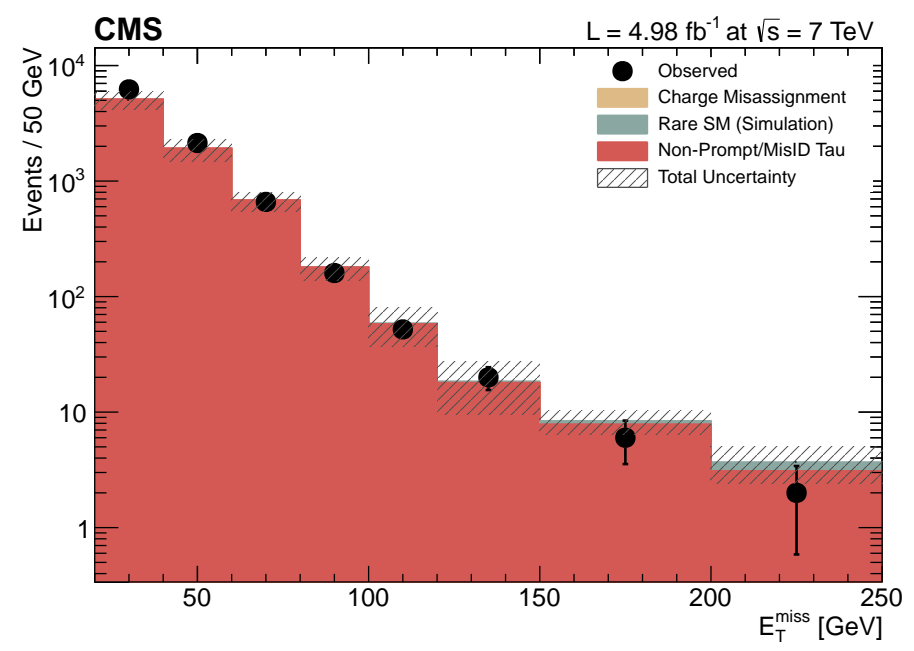

Figure 2: Validation of the background evaluation methods through the comparison between background prediction and observations.

\section{Results}

In table 3 the result in the signal region is shown. No excess of events is seen in the observation compared with the prediction, i.e. $3.5 \pm 0.5$ (stat) \pm 1.5 (syst) events are predicted, while 2 events are observed. Since no excess of events is seen in the observed yield, a $95 \%$ CL upper limit is set. In figure 3 the $95 \%$ CL upper limit on chargino-neutralino production cross section times branching fraction is shown in a plane of the two free parameters of the model: the masses of the chargino and of the neutralino, $m_{\tilde{\chi}_{1}^{ \pm}}$and $m_{\tilde{\chi}_{2}^{0}}$. The other two masses of the new particles that are present in this model are fixed to the following values: $m_{\chi_{2}^{0}}$ is taken equal to the chargino mass, while for 


\begin{tabular}{|c|c|c|}
\hline Channel & Prediction & Observation \\
\hline \hline$e \tau$ & $1.6 \pm 0.3($ stat $) \pm 1.1($ syst $)$ & $\mathbf{1}$ \\
\hline$\mu \tau$ & $1.9 \pm 0.3($ stat $) \pm 1.0($ syst $)$ & $\mathbf{1}$ \\
\hline$\tau \tau$ & $0.0 \pm 0.0($ stat $) \pm 0.0($ syst $)$ & $\mathbf{0}$ \\
\hline \hline Total & $\mathbf{3 . 5} \pm \mathbf{0 . 5}($ stat $) \pm \mathbf{1 . 5}($ syst $)$ & $\mathbf{2}$ \\
\hline
\end{tabular}

Table 1: Comparison between the background predictions and the observations in the signal region.

the slepton mass two different values are taken, parametrized by $m_{\tilde{l}}=x \cdot m_{\tilde{\chi}_{1}^{ \pm}}+(1-x) \cdot m_{\tilde{\chi_{1}^{0}}}$, with $x=0.25$ and 0.75. In figure 3, both the limits from the SSDL and the three leptons plus missing transverse energy searches are shown. The combined results probe charginos/neutralinos up to about $400 \mathrm{GeV}$.
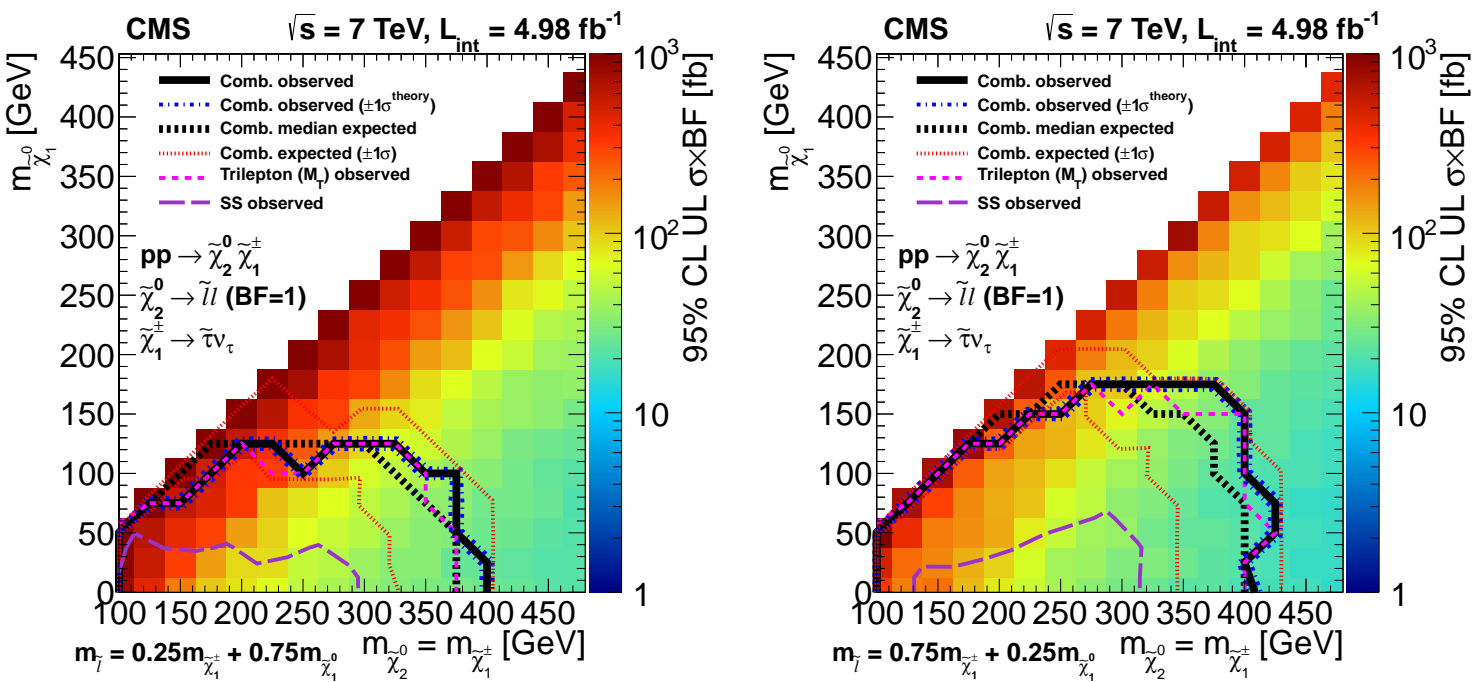

Figure 3: The 95\% CL upper limit on chargino-neutralino production cross section times branching fraction in the $m_{\tilde{\chi_{1}^{0}}}$ versus $m_{{\tilde{\chi_{2}^{0}}}_{0}}\left(=m_{\chi_{1}^{ \pm}}\right)$plane. The SSDL results are combined with the three-lepton search results. The two sub-figures are the results for two different intermediate masses $m_{\tilde{\ell}}$

\section{Conclusions}

Supersymmetry is one of the favored extension of the SM at higher energy. Early search at CMS focused on the search of strongly interacting particles that can be produced with large cross sections, so that models with electroweak production of sparticles could have eluded detection in early searches. Here a search for the model known as chargino-neutralino pair production, i.e. for one important process of the electroweak sector of supersymmetry is shown. No excess above the standard model expectations is observed, so that the results are used to exclude a range of chargino and neutralino masses till about $400 \mathrm{GeV}$. 


\section{References}

[1] CMS Collaboration, Search for electroweak production of charginos and neutralinos using leptonic final states in pp collisions at 7 TeV, JHEP 11 (2012) 147, doi:10.1007/JHEP11(2012)147.

[2] CMS Collaboration, The CMS experiment at the CERN LHC, JINST 03 (2008) S08003, doi:10.1088/1748-0221/3/08/S08004.

[3] R. Barnett, J. Gunion, and H. Haber, Discovering supersymmetry with like sign dileptons, Phys. Lett. B 315 (1993) 349, doi:10.1016/0370-2693(93)91623-U.

[4] M. Guchait and D. P. Roy, Like sign dilepton signature for gluino production at the CERN LHC including top quark and Higgs boson effects, Phys. Rev. D 52 (1995) 133, doi:10.1103/PhysRevD.52.133.

[5] O. Panella, M. Cannoni, C. Carimalo and Y. N. Srivastava, Signals of heavy Majorana neutrinos at hadron colliders, Physical Review D 65 (2002) 035005, doi:10.1103/PhysRevD.65.035005.

[6] CMS Collaboration, Search for a Heavy Bottom-like Quark in pp Collisions at $\sqrt{s}=7$ TeV, Phys. Lett. B 701 (2011), 204-223, doi:10.1016/j.physletb.2011.05.074

[7] CMS Collaboration, Search for New Physics with Same-Sign Isolated Dilepton Events with Jets and Missing Transverse Energy, Phys. Rev. Lett. 109, 071803 (2012), doi:10.1103/PhysRevLett.109.071803.

[8] Johan Alwall, Philip Schuster and Natalia Toro, Simplified Models for a First Characterization of New Physics at the LHC, Phys. Rev. D 79 (2009) 075020, doi:10.1103/PhysRevD.79.075020. 